\title{
Asymptotic Behavior of an SI Epidemic Model with Pulse Removal
}

\author{
K.M. Fuhrman, I.G. Lauko and G.A. Pinter \\ University of Wisconsin-Milwaukee, Department of Mathematical Sciences, \\ Milwaukee, WI 53201-0413
}

\begin{abstract}
In this paper we discuss an SI epidemic model with pulse removal from the infective class at fixed time intervals with both exponential and logistic type underlying population dynamics. This model has a significance when dealing with animal diseases with no recovery, or when we consider isolation in human diseases. We provide a rigorous analysis of the asymptotic behavior of the percentage of infected individuals, the total number of infected individuals, and the total population in our model. We show that periodic removal/isolation is a feasible strategy to control the spread of the disease.
\end{abstract}

Key words: Epidemiology, Asymptotic behavior, Impulsive differential equations

\section{Introduction}

Mathematical methods are widely used for understanding mechanisms in the spread of infectious diseases. Together with computer simulations, epidemic models are very useful in explaining and testing various theories, determining key parameters, and finding the effects of changes in the parameter values. The high interest can be explained by the economic and societal effects of various known epidemic diseases and the emergence of new viruses and infections. Another important motivation for the development of this field is the evaluation of various vaccination/control strategies for human as well as animal diseases. The study of epidemics among animals has gained a lot of attention in recent years with major outbreaks of the mad cow and hoof-andmouth diseases together with the spread of chronic wasting disease in the US $[9,10]$.

Infectious animal diseases have a great effect in the ecology and management of wildlife and domestic animals. Sometimes the diseases among animals pose 


\section{Report Documentation Page}

Form Approved

OMB No. 0704-0188

Public reporting burden for the collection of information is estimated to average 1 hour per response, including the time for reviewing instructions, searching existing data sources, gathering and maintaining the data needed, and completing and reviewing the collection of information. Send comments regarding this burden estimate or any other aspect of this collection of information,

including suggestions for reducing this burden, to Washington Headquarters Services, Directorate for Information Operations and Reports, 1215 Jefferson Davis Highway, Suite 1204, Arlington

VA 22202-4302. Respondents should be aware that notwithstanding any other provision of law, no person shall be subject to a penalty for failing to comply with a collection of information if it

does not display a currently valid OMB control number.

1. REPORT DATE

2006

4. TITLE AND SUBTITLE

Asymptotic Behavior of an SI Epidemic Model with Pulse Removal

6. AUTHOR(S)

7. PERFORMING ORGANIZATION NAME(S) AND ADDRESS(ES)

University of Wisconsin-Milwaukee,Department of Mathematical Sciences,Milwaukee,WI,53201-0413

9. SPONSORING/MONITORING AGENCY NAME(S) AND ADDRESS(ES)

3. DATES COVERED

00-00-2006 to 00-00-2006

5a. CONTRACT NUMBER

5b. GRANT NUMBER

5c. PROGRAM ELEMENT NUMBER

5d. PROJECT NUMBER

5e. TASK NUMBER

5f. WORK UNIT NUMBER

8. PERFORMING ORGANIZATION REPORT NUMBER

10. SPONSOR/MONITOR'S ACRONYM(S)

11. SPONSOR/MONITOR'S REPORT NUMBER(S)

12. DISTRIBUTION/AVAILABILITY STATEMENT

Approved for public release; distribution unlimited

13. SUPPLEMENTARY NOTES

14. ABSTRACT

see report

15. SUBJECT TERMS

16. SECURITY CLASSIFICATION OF:

a. REPORT

unclassified b. ABSTRACT

unclassified c. THIS PAGE

unclassified
17. LIMITATION OF ABSTRACT
18. NUMBER

OF PAGES

17 19a. NAME OF

RESPONSIBLE PERSON 
a great threat to public health. When there is an epidemic outbreak among animals, a common way to control it is by destroying a fraction of the infected population. In [10] Heesterbeek and Roberts considered a model with a fixed culling rate $\gamma$ and showed its effect on the dynamics of the infected population. However, the constant removal strategy assumes continuous culling at the same rate, which is not a realistic approach. We propose a new model that assumes that a fraction of the infected population is being removed at certain fixed points in time and not continuously.

The effects of pulse removal have been studied before in relation to epidemic models while analyzing vaccination methods $[1,2,7,8]$, where a fixed portion of the susceptible population was removed (i.e. vaccinated) at fixed time intervals. It was shown that the infection free solution can be obtained with lower levels of vaccination compared to the constant vaccination strategy. Our goal is to show that pulse removal from the infected class, which can model the actual destruction of a portion of the population or isolation from the rest of the population, is a feasible strategy to control the disease. The control objective is to drive the number of infected individuals to zero while stabilizing the population size or growth rate.

In section 2 we consider an SI model with exponential underlying dynamics for the total population. The inclusion of pulse removal transforms the system into a system of impulsive differential equations [4,5]. We establish a threshold value $p_{1}^{*}$ for the removed fraction $p$ such that for $p<p_{1}^{*}$ the fraction of infected individuals tends to a positive periodic function, while for $p>p_{1}^{*}$ this fraction tends to 0 . However, the fact that the fraction of infected individuals tends to 0 does not guarantee the eradication of the disease in a growing population. Thus, it is of interest that we establish another threshold value, $p_{2}^{*}$, which has the property that for $p>p_{2}^{*}$ the number of infected individuals tends to 0 , while the population grows. This case realizes the control objective. We also provide a detailed analysis of the asymptotic behavior of the total population for $p<p_{2}^{*}$, and illustrate the different possible cases.

In section 3 we carry out an analogous study for an SI model with logistic dynamics for the total population, which may provide a more realistic approach $[3,6,11,12]$.

\section{SI Model with Pulse Removal}

We consider a common SI model with exponential dynamics for the total population. When pulse removal is introduced in this model, we obtain the following system: 


$$
\begin{aligned}
& \frac{d S}{d t}=b N-d S-\frac{\beta S I}{N} \\
& \frac{d I}{d t}=-(d+\alpha) I+\frac{\beta S I}{N}-p \sum_{n=1}^{\infty} I\left(n T^{-}\right) \delta(t-n T) \\
& S+I=N
\end{aligned}
$$

Here $I(t) \geq 0, S(t) \geq 0$ denote the size of the infected and susceptible population classes, respectively, and $N(t)$ is the total population at time $t \geq 0$. $b$ is the birth rate, $d$ is the death rate, while $\beta$ denotes the contact rate and $\alpha$ is an added death rate due to the disease. We consider the case when the natural birth rate $b$ is greater then the death rate $d$. In a population that is dying out naturally, that is, if $d>b$, the removal does not affect the asymptotic behavior of the system. Note that (1)-(3) implies that

$$
\frac{d N}{d t}=(b-d) N-\alpha I-p \sum_{n=1}^{\infty} I\left(n T^{-}\right) \delta(t-n T)
$$

In our proposed model, the removal is applied as an impulse whenever $t=n T$, $n=1,2, \ldots$ The fraction $p$ of the infective individuals is being removed from the class, that is, $I(t)$ decreases with proportion $p$ whenever $t=n T$. We will use the notation

$$
I\left(n T^{-}\right)=\lim _{t \rightarrow n T^{-}} I(t) \text { and } I\left(n T^{+}\right)=\lim _{t \rightarrow n T^{+}} I(t)
$$

to indicate the different limits of the solution from left and right at these points. The sequence of impulses creates jump discontinuities in $I(t)$, which decreases with proportion $p$ whenever $t=n T$. We start the analysis by translating the model for infectious and susceptible fractions of the population.

\subsection{Susceptible and Infective Individuals as Fractions of Population}

We translate the given system into a model describing the behavior of $s(t)=$ $\frac{S(t)}{N(t)}, i(t)=\frac{I(t)}{N(t)}$. Now, $s(t)$ and $i(t)$ represent the percentage or fraction of the population that are susceptible and infective, respectively.

Thus the system (1)-(3) becomes:

$$
\begin{aligned}
& \frac{d s}{d t}=b-b s-(\beta-\alpha) s i+\sum_{n=1}^{\infty} p \frac{i\left(n T^{-}\right) s\left(n T^{-}\right)}{1-p i\left(n T^{-}\right)} \delta(t-n T) \\
& \frac{d i}{d t}=-(b+\alpha) i+\beta s i+\alpha i^{2}-\sum_{n=1}^{\infty} p \frac{i\left(n T^{-}\right)\left(1-i\left(n T^{-}\right)\right)}{1-p i\left(n T^{-}\right)} \delta(t-n T)
\end{aligned}
$$


with feasibility set $\mathcal{D}=\{(s, i) \mid s \geq 0, i \geq 0, s+i=1\}$, and we define $\mathcal{D}_{1}=\mathcal{D}-\{(1,0)\}$. We note that the system is well-posed epidemiologically, that is, if the initial conditions $i_{0}$ and $s_{0}$ are in the feasibility region, then $s(t)$ and $i(t)$ will stay in $\mathcal{D}$ for all $t \geq 0$.

Theorem 1: Consider the system of equations (6)-(7) with $b>0, \beta-(b+$ $\alpha)>0$ and all other parameters non-negative. The following holds with $p_{1}^{*}=$ $1-e^{-(\beta-b-\alpha) T}$ :

(1) If $p>p_{1}^{*}$ then the disease free equilibrium $(s, i)=(1,0)$ always exists and it is globally asymptotically stable in the feasibility set $\mathcal{D}$.

(2) If $p<p_{1}^{*}$ then there exists a unique endemic periodic solution $\left(i^{*}, s^{*}\right)$ and this solution is globally asymptotically stable in $\mathcal{D}_{1}$.

Proof: Since $s(t)=1-i(t)$, it is sufficient to consider the initial value problem

$$
\begin{aligned}
\frac{d i}{d t} & =-(b+\alpha) i+\beta(1-i) i+\alpha i^{2} \\
& -\sum_{n=1}^{\infty} p \frac{i\left(n T^{-}\right)\left(1-i\left(n T^{-}\right)\right)}{1-p i\left(n T^{-}\right)} \delta(t-n T) \\
i(0) & =i_{0}>0 .
\end{aligned}
$$

On an interval $[n T,(n+1) T](8)$ is a logistic equation in $i$ and it can be explicitly solved. We note that the assumption $\beta>(b+\alpha)$ guarantees that the equation (8) without the pulse removal has two stationary solutions, $i_{1}(t) \equiv 0$ and $i_{2}(t) \equiv \frac{\beta-(b+\alpha)}{\beta-\alpha}$, with $0<i_{2}(t)<1$. The trivial solution $i_{1}(t)$ is unstable, while $i_{2}(t)$ is asymptotically stable. If $\beta<(b+\alpha)$, then there are two cases:

a) If $\beta-\alpha>0$, then $i_{2}(t)<0$, and in this case $i_{1}(t) \equiv 0$ is asymptotically stable, in particular, for any $i(0)=i_{0}, 0<i_{0} \leq 1, i(t) \rightarrow 0$ as $t \rightarrow \infty$, where $i$ is the solution of (8) without the pulse removal. Thus the fraction of infected individuals goes to zero naturally without any controlling mechanism.

b) If $\beta-\alpha<0$, then $i_{2}(t)>1$, and we again have that for any $0<i_{0} \leq 1$, $i(t) \rightarrow 0$ as $t \rightarrow \infty$.

We also remark that the assumption $\beta>(b+\alpha)$ is realistic in epidemiological applications. Solving $(8)$ on an interval $[n T,(n+1) T]$ we obtain

$$
G(t)=\frac{i\left(n T^{+}\right) e^{(\beta-b-\alpha)(t-n T)}}{1+\frac{i\left(n T^{+}\right)(\beta-\alpha)}{\beta-b-\alpha}\left(e^{(\beta-b-\alpha)(t-n T)}-1\right)} .
$$

At the removal points we have 


$$
i\left((n+1) T^{+}\right)=\frac{(1-p) G\left((n+1) T^{-}\right)}{1-p G\left((n+1) T^{-}\right)} .
$$

Under our assumptions this solution is well-defined and exists for all $t \geq 0$.

Now, we consider the sequence of the "initial" values for each interval between removals at $i\left(n T^{+}\right)$, where $n=1,2, \ldots$. We let $i\left(n T^{+}\right)=i_{n}$ and deduce a stroboscopic map $F$ that satisfies

$$
i_{n+1}=F\left(i_{n}\right)
$$

The map $F$ determines the value of $i(t)$ immediately after each removal at discrete times $t=n T$. Then,

$$
\begin{aligned}
i_{n+1}=F\left(i_{n}\right) & =\frac{(1-p) i\left((n+1) T^{-}\right)}{1-p i\left((n+1) T^{-}\right)} \\
& =\frac{(1-p) i_{n} e^{(\beta-b-\alpha) T}}{1+\frac{\beta-\alpha}{\beta-b-\alpha} i_{n}\left(e^{(\beta-b-\alpha) T}-1\right)-p i_{n} e^{(\beta-b-\alpha) T}}
\end{aligned}
$$

If $p \neq \frac{(\beta-\alpha) e^{(\beta-b-\alpha) T}-1}{(\beta-b-\alpha) e^{(\beta-b-\alpha) T}}$, the map $F$ has two fixed points

$$
i_{1}^{*}=0 \text { and } i_{2}^{*}=\frac{(1-p) e^{(\beta-b-\alpha) T}-1}{\frac{\beta-\alpha}{\beta-b-\alpha}\left(e^{(\beta-b-\alpha) T}-1\right)-p e^{(\beta-b-\alpha) T}} .
$$

We analyze $i_{2}^{*}$ as a function of $p$. Figure 1 illustrates the general behavior of

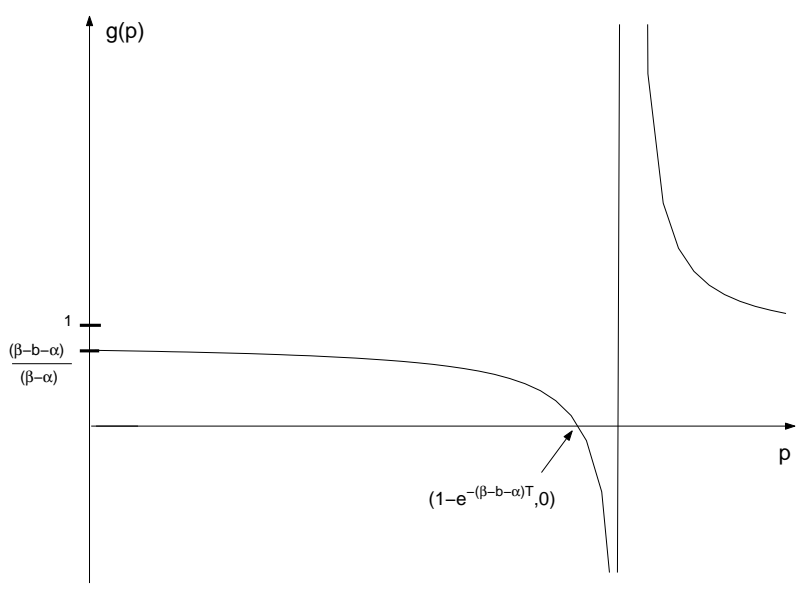

Fig. 1.

General behavior of the function $g(p)$

the function $i_{2}^{*}=g(p)$. For $p=0$, we have $g(0)=\frac{\beta-b-\alpha}{\beta-\alpha}$. The function has 
an asymptote at $p_{a}=\frac{(\beta-\alpha) e^{(\beta-b-\alpha) T}-1}{(\beta-b-\alpha) e^{(\beta-b-\alpha) T}}$, which may or may not be less than 1. The function is always decreasing for $p<p_{a}$, and, $\lim _{p \rightarrow p_{a}^{-}} g(p)=-\infty$. The zero of the function is at $p=p_{1}^{*}=1-e^{-(\beta-b-\alpha) T}<p_{a}$. If $p>p_{1}^{*}$ then $g(p)<0$ or $g(p)>1$, which brings it outside the feasibility region $\mathcal{D}$. Finding the derivative of $F$ at the two fixed points will determine the stability of those fixed points. We obtain

$$
\frac{d F\left(i_{n}\right)}{d i_{n}}=\frac{e^{(\beta-b-\alpha) T}(1-p)}{\left(1+\frac{\beta-\alpha}{\beta-b-\alpha} i_{n}\left(e^{(\beta-b-\alpha) T}-1\right)-p i_{n} e^{(\beta-b-\alpha) T}\right)^{2}} .
$$

The derivative at the fixed points yields

$$
\begin{aligned}
& \left.\frac{d F\left(i_{n}\right)}{d i_{n}}\right|_{i_{n}=i_{1}^{*}}=(1-p) e^{(\beta-b-\alpha) T} \\
& \left.\frac{d F\left(i_{n}\right)}{d i_{n}}\right|_{i_{n}=i_{2}^{*}}=\frac{1}{(1-p) e^{(\beta-b-\alpha) T}}
\end{aligned}
$$

In order for $i_{1}^{*}=0$ to be locally asymptotically stable we make

$$
\left.\frac{d F\left(i_{n}\right)}{d i}\right|_{i_{n}=i_{1}^{*}}<1
$$

which implies

$$
p>1-e^{-(\beta-b-\alpha) T} .
$$

If condition (12) holds, then the other fixed point $i_{2}^{*}$ is not in the feasibility set, that is, either $i_{2}^{*}<0$ of $i_{2}^{*}>1$. The above argument also shows that in the case when $p<1-e^{-(\beta-b-\alpha) T}, i_{2}^{*}$ is locally asymptotically stable, while $i_{1}^{*}$ is unstable.

Now, we show the global stability of the fixed points. Since,

$$
\frac{d F\left(i_{n}\right)}{d i_{n}}=\frac{e^{(\beta-b-\alpha) T}(1-p)}{\left(1+\frac{\beta-\alpha}{\beta-b-\alpha} i_{n}\left(e^{(\beta-b-\alpha) T}-1\right)-p i_{n} e^{(\beta-b-\alpha) T}\right)^{2}}>0
$$

for any $0 \leq i_{n} \leq 1$, we have that $\left\{F^{n}\left(i_{0}\right)\right\}=\left\{i_{0}, F\left(i_{0}\right), F\left(F\left(i_{0}\right)\right), \ldots\right\}$ is a monotone sequence for any initial condition $i_{0}$. This sequence is bounded between 0 and 1 by the well-posedness of our system. Therefore, we have obtained a monotone bounded sequence which has to converge to some limit $L$. We proceed by finding this limit. 


$$
L=\lim _{n \rightarrow \infty} F^{n}\left(i_{0}\right)=\frac{(1-p) L e^{(\beta-b-\alpha) T}}{1+\frac{\beta-\alpha}{\beta-b-\alpha} L\left(e^{(\beta-b-\alpha) T}-1\right)-p L e^{(\beta-b-\alpha) T}} .
$$

Solving for $L$ we obtain the following result

$$
L_{1}=i_{1}^{*}=0, \quad L_{2}=i_{2}^{*}=\frac{(1-p) e^{(\beta-b-\alpha) T}-1}{\frac{\beta-\alpha}{\beta-b-\alpha}\left(e^{(\beta-b-\alpha) T}-1\right)-p e^{(\beta-b-\alpha) T}} .
$$

If condition (12) holds then $L_{2} \notin \mathcal{D}_{1}$, so $L_{1}=0$ is the limit of $\left\{F^{n}\left(i_{0}\right)\right\}$ for any $i_{0}$, and thus it is a globally asymptotically stable fixed point of $F\left(i_{n}\right)$. Hence in this case $i(t) \rightarrow 0$ as $t \rightarrow \infty$ for any initial condition $i_{0} \in \mathcal{D}$. If $p<1-e^{-(\beta-b-\alpha) T}$, then $L_{2}$ is the limit of $\left\{F^{n}\left(i_{0}\right)\right\}$ and thus $L_{2}$ is globally asymptotically stable in $\mathcal{D}_{1}$. Then there is a unique endemic periodic solution $i_{2}^{*}(t)=i(t)$ with $i_{0}=L_{2}$. This periodic endemic solution is globally asymptotically stable, meaning that $\left|i(t)-i_{2}^{*}(t)\right| \rightarrow 0$ as $t \rightarrow \infty$ for any $i(t)$ which solves (1)-(3). This concludes the proof of Theorem 1 .

\subsection{Total Number of Infective Individuals}

Now, we generalize the result from the previous section to study the behavior of the total number of infective individuals and the total population. We go back to the system of equations (1)-(3).

Theorem 2: Consider the system of equations (1)-(3) under the assumption that $b>d>0, \beta-(\alpha+b)>0$ and all other parameters non-negative. Then with $p_{2}^{*}=1-e^{-(\beta-d-\alpha) T}$ the following hold:

(1) If $p>1-e^{-(\beta-d-\alpha) T}$ the total infected population has the asymptotic behavior $I(t) \rightarrow 0$.

(2) If $1-e^{-(\beta-b-\alpha) T}<p<1-e^{-(\beta-d-\alpha) T}$ the total infected population has the asymptotic behavior $I(t) \rightarrow \infty$.

Proof: First, we consider the case when $p>1-e^{-(\beta-b-\alpha) T}$, that is, the fraction of infected population goes to 0 . By the previous theorem, we know that under this condition, $s(t)=\frac{S(t)}{N(t)} \rightarrow 1$. Then for any $\varepsilon>0$ there exists $t_{0}$ such that $1-\varepsilon<\frac{S(t)}{N(t)} \leq 1$ for $t \geq t_{0}$. Let $t_{0}=\left(n_{0} T\right)^{+}$. Consider,

$$
\frac{d I}{d t}=-(d+\alpha) I+\frac{\beta S I}{N}-p \sum_{n=1}^{\infty} I\left(n T^{-}\right) \delta\left(t-t_{0}-n T\right), \quad t>t_{0}
$$

with $I\left(t_{0}\right)=I_{0}$. Under the assumption that $1-\varepsilon<\frac{S}{N} \leq 1$ for $t \geq t_{0}$, we introduce 


$$
\frac{d \hat{I}}{d t}=-(d+\alpha) \hat{I}+\beta(1-\varepsilon) \hat{I}-p \sum_{n=1}^{\infty} \hat{I}\left(n T^{-}\right) \delta\left(t-t_{0}-n T\right)
$$

with $\hat{I}\left(t_{0}\right)=I_{0}$.

Then, $I(t)>\hat{I}(t)$ for all $t>t_{0}$ and

$$
\hat{I}(t)=I_{0}\left[(1-p) e^{(\beta(1-\varepsilon)-d-\alpha) T}\right]^{n} e^{(\beta(1-\varepsilon)-d-\alpha)(t-n T)},
$$

for $n T \leq t \leq(n+1) T, n \geq n_{0}$.

Thus, the sequence of "initial" values at $\hat{I}\left(n T^{+}\right)$where $n=n_{0}, n_{0}+1, n_{0}+2 \ldots$ forms a geometric progression, which converges to 0 if $(1-p) e^{(\beta(1-\varepsilon)-d-\alpha) T}<1$ and grows without bounds in the reverse case.

Therefore, we conclude that if $p<1-e^{-(\beta(1-\varepsilon)-d-\alpha) T}$ then $\hat{I}(t) \rightarrow \infty$ as $t \rightarrow \infty$. Since $I(t)>\hat{I}(t)$ for $t \geq t_{0}$ and $\varepsilon>0$ is arbitrary, we have $I(t) \rightarrow \infty$ as $t \rightarrow \infty$ if $p<1-e^{-(\beta-d-\alpha) T}$.

Next let us consider

$$
\begin{aligned}
& \frac{d \tilde{I}}{d t}=-(d+\alpha) \tilde{I}+\beta \tilde{I}-p \sum_{n=1}^{\infty} \tilde{I}\left(n T^{-}\right) \delta\left(t-t_{0}-n T\right) \\
& \text { with } \tilde{I}\left(t_{0}\right)=I_{0} .
\end{aligned}
$$

In this case, we have $I(t) \leq \tilde{I}(t)$. By similar analysis we obtain that for $p>1-e^{-(\beta-d-\alpha) T}$ we have $\tilde{I}(t) \rightarrow 0$ as $t \rightarrow \infty$. Since $0 \leq I(t) \leq \tilde{I}(t)$, we have that $I(t) \rightarrow 0$ as well, which concludes the proof of Theorem (6.2).

We note that the assumption $b>d$ guarantees that the threshold $p_{2}^{*}$ for $I(t)$ is greater than $p_{1}^{*}$.

Now, we consider the behavior of $I(t)$ when $p<1-e^{-(\beta-b-\alpha) T}$. In this case, $i(t)$ approaches an endemic periodic solution $i_{2}^{*}(t)$, for which $i_{2}^{*}\left(n T^{+}\right) \equiv i_{2}^{*}$, where $i_{2}^{*}$ is the nonzero fixed point of the map $F$ in (10). We introduce

$$
H(p)=(1-p) e^{(\beta-d-\alpha) T}\left(\frac{\beta-\alpha}{\beta-b-\alpha} i_{2}^{*}\left(e^{(\beta-b-\alpha) T}-1\right)+1\right)^{\frac{-\beta}{\beta-\alpha}}
$$

where, $i_{2}^{*}$ is given by (11). The definition of $H(t)$ is motivated later in the proof of the following theorem, which describes the dynamics of $I(t)$ under the conditions $p<1-e^{-(\beta-b-\alpha) T}$ and $\beta>(b+\alpha)$. 
Theorem 3: Consider the system of equations (1)-(3) with $b>d>0, \beta>$ $(b+\alpha)$ and all other parameters non-negative. Let $p<1-e^{-(\beta-b-\alpha) T}$. In this case we have:

(1) If $H(p)<1$, then the total infected population has the asymptotic behavior $I(t) \rightarrow 0$.

(2) If $H(p)>1$, the total infected population has the asymptotic behavior $I(t) \rightarrow \infty$.

Proof: From (2) we have that:

$$
\frac{d I}{d t}=-(d+\alpha) I+\frac{\beta S I}{N}-p \sum_{n=1}^{\infty} I\left(n T^{-}\right) \delta(t-n T) .
$$

Substituting $s(t)=S(t) / N(t)$ we obtain

$$
\frac{d I}{d t}=\beta s(t) I-(\alpha+d) I-p \sum_{n=1}^{\infty} I\left(n T^{-}\right) \delta(t-n T)
$$

then

$$
\frac{d I}{d t}=(\beta(1-i(t))-(\alpha+d)) I-p \sum_{n=1}^{\infty} I\left(n T^{-}\right) \delta(t-n T) .
$$

We solve this equation for $(n-1) T \leq t<n T$ and obtain

$$
I(t)=I_{0} e^{\int_{0}^{t-(n-1) T} \beta(1-i(\tau))-(\alpha+d) d \tau}, \quad \text { where } I_{0}=I\left((n-1) T^{+}\right)
$$

From Theorem 1, we know that $i(t)$ approaches an endemic periodic solution $i_{2}^{*}(t)$, with $i_{2}^{*}\left(n T^{+}\right)=i_{2}^{*}$. Thus, $i(\tau)$ is approximately $\frac{i_{2}^{*} e^{(\beta-b-\alpha) \tau}}{1+\frac{\beta-\alpha}{\beta-b-\alpha} i_{2}^{*}\left(e^{(\beta-b-\alpha) \tau}-1\right)}$, $(n-1) T \leq \tau<n T$ for large $n$.

We consider $I\left(n T^{+}\right)$, the number of infective individuals right after the removals. Then we obtain the following:

$$
\begin{aligned}
I\left(n T^{+}\right) & =(1-p) I_{0} e^{(\beta-d-\alpha) T} e^{\int_{0}^{T}-\beta \frac{i_{2}^{*}(\beta-b-\alpha) \tau}{\frac{\beta-\alpha}{\beta-b-\alpha} i_{2}^{*} e^{(\beta-b-\alpha) \tau}+\left(1-\frac{\beta-\alpha}{\beta-b-\alpha} i_{2}^{*}\right)} d \tau} \\
& =(1-p) I_{0} e^{(\beta-d-\alpha) T}\left(\frac{\beta-\alpha}{\beta-b-\alpha} i_{2}^{*}\left(e^{(\beta-b-\alpha) T}-1\right)+1\right)^{\frac{-\beta}{\beta-\alpha}}
\end{aligned}
$$

Hence, the function 


$$
H(p)=(1-p) e^{(\beta-d-\alpha) T}\left(\frac{\beta-\alpha}{\beta-b-\alpha} i_{2}^{*}\left(e^{(\beta-b-\alpha) T}-1\right)+1\right)^{\frac{-\beta}{\beta-\alpha}}
$$

determines the behavior of $I(t)$. The above calculation shows that $I\left(n T^{+}\right)=$ $I_{0} H(p)$, where $I_{0}=I\left((n-1) T^{+}\right)$. Repeated application of this relationship yields

$$
I\left(m T^{+}\right)=I_{0}(H(p))^{(m-(n-1))} m \geq n
$$

Hence, if $H(p)>1$ for a particular $p, p<1-e^{-(\beta-b-\alpha) T}$, then $I(t) \rightarrow \infty$ as $t \rightarrow \infty$ for this value of $p$; and if $H(p)<1$ then $I(t) \rightarrow 0$.

In the next section we show the examples for different possible behaviors of this function.

\subsection{Total Population}

We presented a complete analysis of the behavior of the fraction of infective individuals $i(t)$ and the total infective population $I(t)$. Using this information we can determine the dynamics of the total population $N(t)$ for various values of $p$. The dynamics of the solutions of (1)-(3) can be summarized as follows for various values of the parameter $p$.

\begin{tabular}{|c|r|c|c|}
\hline \multicolumn{1}{|c|}{$\mathrm{p}$} & $\mathrm{i}(\mathrm{t})$ & $\mathrm{I}(\mathrm{t})$ & $\mathrm{N}(\mathrm{t})$ \\
\hline \hline & & & \\
$p<p_{1}^{*}$ and $H(p)<1$ & $i \rightarrow$ endemic periodic solution & $I \rightarrow 0$ & $N \rightarrow 0$ \\
$p<p_{1}^{*}$ and $H(p)>1$ & $i \rightarrow$ endemic periodic solution & $I \rightarrow \infty$ & $N \rightarrow \infty$ \\
$p_{1}^{*}<p<p_{2}^{*}$ & $i \rightarrow 0$ & $I \rightarrow \infty$ & $N \rightarrow \infty$ \\
$p>p_{2}^{*}$ & $i \rightarrow 0$ & $I \rightarrow 0$ & $N \rightarrow \infty$ \\
\hline
\end{tabular}

Table 1: Dynamics of solutions of (1)-(3) for various values of parameter $p$, with $b>d, \beta>(b+\alpha)$

Therefore, we have obtained a complete description of the asymptotic behavior of the solutions to our proposed model.

As noted before, based on the behavior of the function $H(p)$ we can have different scenarios. The following examples depict some interesting cases. We note that the parameter values in the examples are for illustration purposes, 
they do not necessarily represent real-life situations. We use the following notation for the threshold values of $p$ in the interval $[0,1]$

$$
\begin{aligned}
p_{h_{1}} & =\text { value of } p \text { where } H(p)=1 \text { and } H(p) \text { is decreasing, } \\
p_{h_{2}} & =\text { value of } p \text { where } H(p)=1 \text { and } H(p) \text { is increasing, } \\
p_{1}^{*} & =1-e^{-(\beta-b-\alpha) T}, \text { the threshold of the fraction of infected population } i(t), \\
p_{2}^{*} & =1-e^{-(\beta-d-\alpha) T}, \text { the threshold of the total infected population. } I(t)
\end{aligned}
$$

Example 1: We consider the case when first $H(p)<1$ and then $H(p)>1$, hence $p_{h_{1}}$ does not exist in $[0,1]$. This scenario can be described, for example, by the following parameter values: $\beta=2.25, b=0.4, d=0.1, \alpha=$ 0.8 , and $T=1$. The thresholds for this example are as follows: $p_{h_{2}}=$ $.6197, p_{1}^{*}=.650062$, and $p_{2}^{*}=.74076$. First, we graph the case when $p=.5<p_{h_{2}}$. Hence, we should obtain that $i$ converges to the endemic periodic solution, $I \rightarrow 0$ and $N \rightarrow 0$. The results of the numerical simulation for this case are depicted in Figure 2.
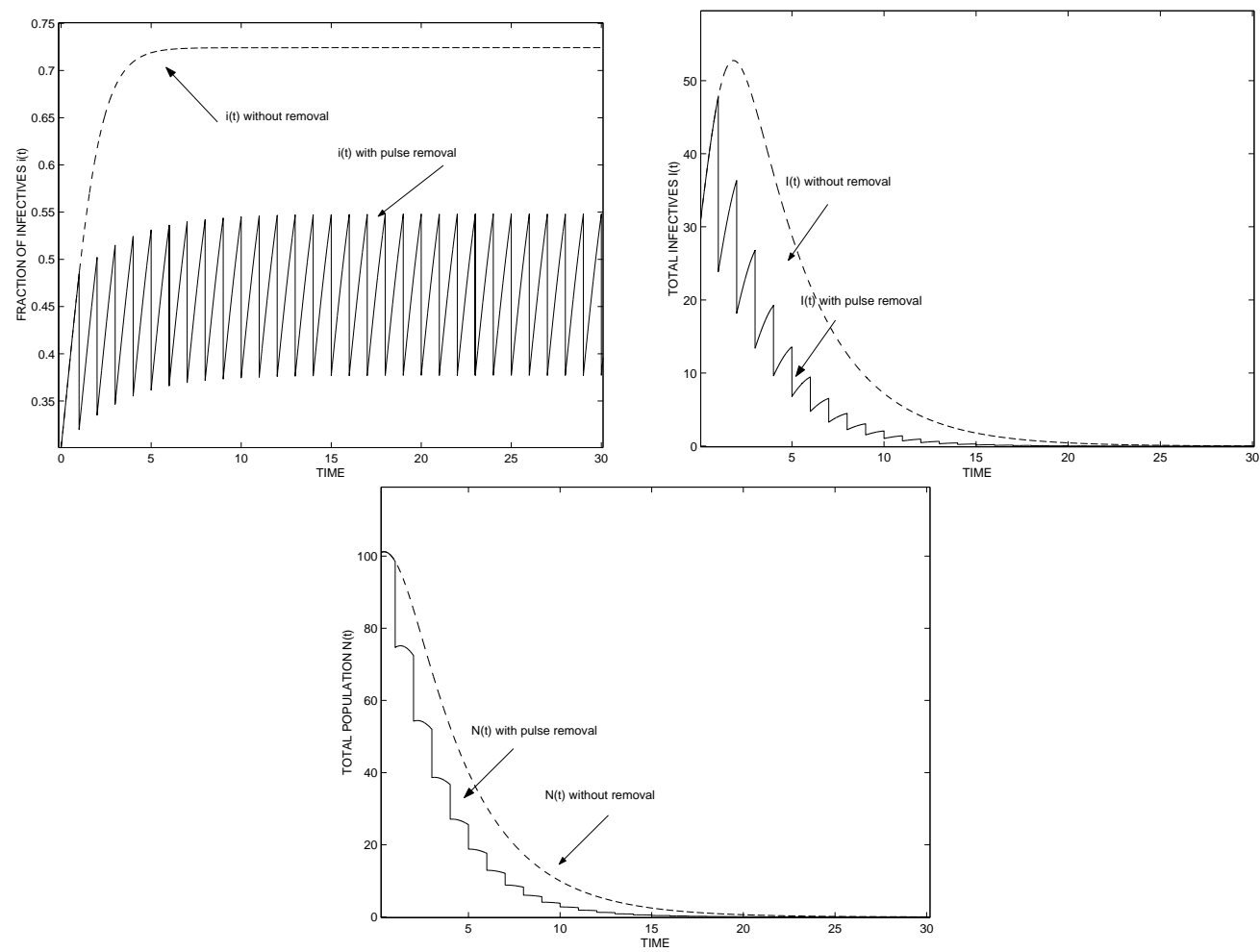

Fig. 2. Example 1 for $p=0.5$ 
If $p_{h_{2}}<p=.63<i_{t h}$ then $i$ converges to the endemic periodic solution, $I \rightarrow \infty$, and $N \rightarrow \infty$.



Fig. 3. Example 1 for $p=0.63$ 
Thus, we can see that the larger pulse removal $p$ in this case has the effect of 'saving' the population in the sense that now $N(t) \rightarrow \infty$ as $t \rightarrow \infty$. At the same time $I(t) \rightarrow \infty$ also, i.e., the disease is sustained in the population as shown in Figure 3.

If $i_{t h}<p=.7<p_{2}^{*}$, then $i \rightarrow 0, I \rightarrow \infty$, and $N \rightarrow \infty$. The larger percentage

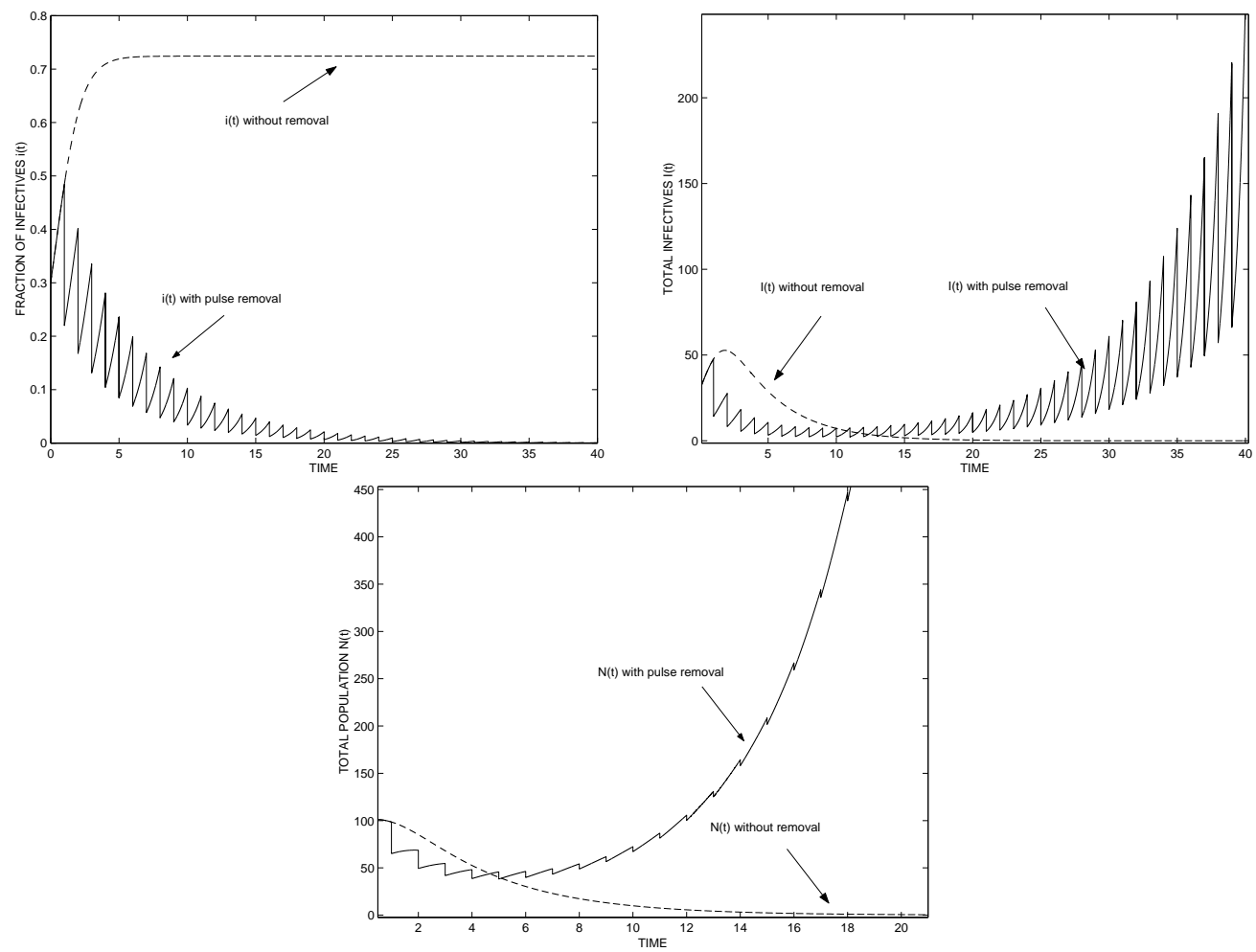

Fig. 4. Example 1 for $p=0.7$

of removed infective individuals now results in the fraction of the infected population $i(t) \rightarrow 0$, while $I(t), N(t) \rightarrow \infty$ (see Figure 4 ).

If $p=0.77>p_{2}^{*}$ then $i \rightarrow 0, I \rightarrow 0$, and $N \rightarrow \infty$. Figure 5 shows that the removal of $77 \%$ of infected individuals drives the total number of infective individuals to zero, while the population is growing without bounds. Thus, with this control strategy the disease is eradicated while population is flourishing. (This is in contrast with the no-control $(p=0)$ case where the population is eradicated together with the disease.)

\section{SI model with logistic dynamics}

In this section we study an SI model with pulse removal that has a logistic underlying dynamics for the total population $S+I$. This is considered a more 


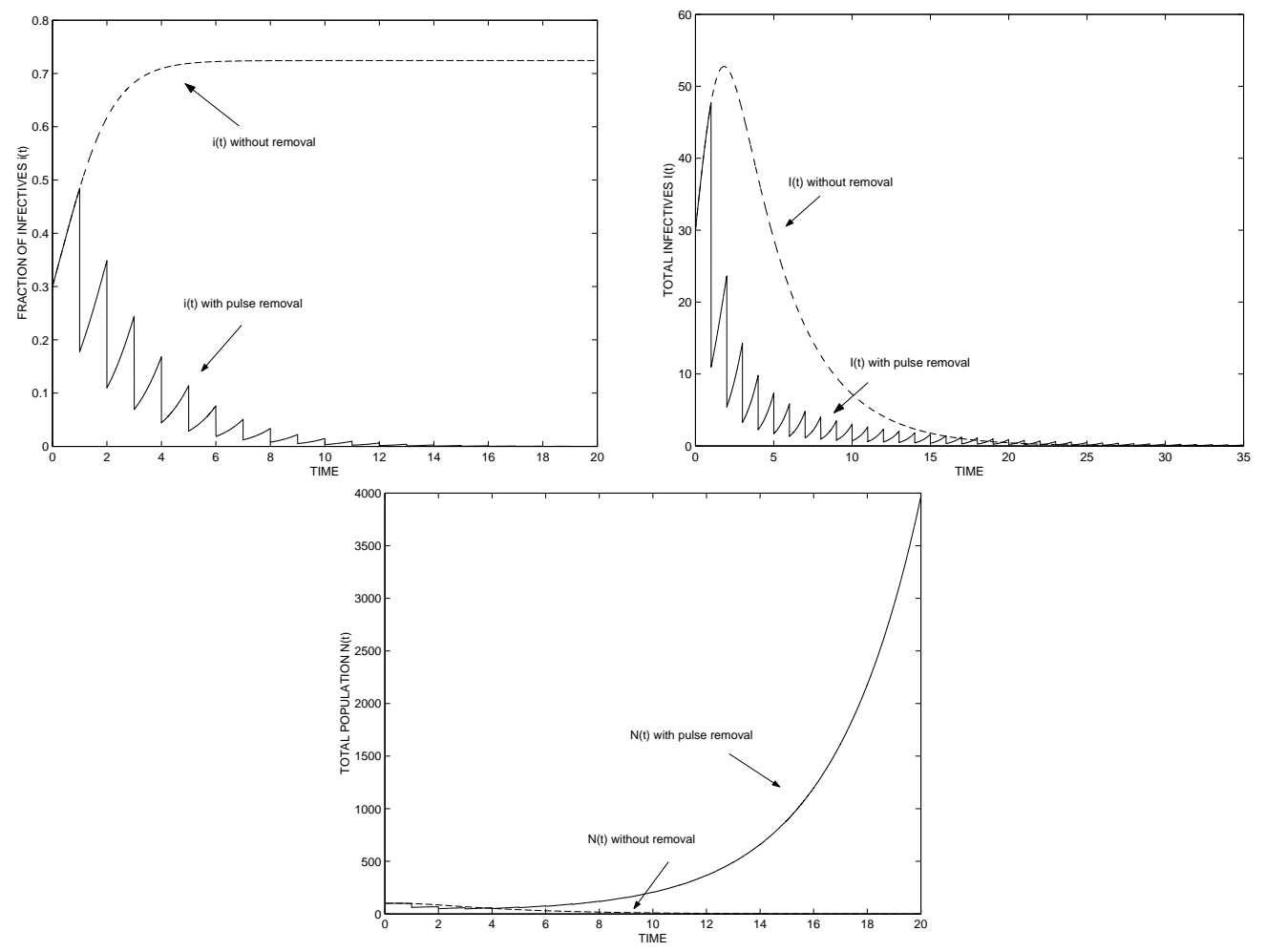

Fig. 5. Example 1 for $p=0.77$

realistic approach than the exponential dynamics especially for longer time intervals. We consider the model

$$
\begin{aligned}
& \frac{d S}{d t}=b N-d(1+r N) S-\frac{\beta S I}{N} \\
& \frac{d I}{d t}=-(d(1+r N)+\alpha) I+\frac{\beta S I}{N}-p \sum_{n=1}^{\infty} I\left(n T^{-}\right) \delta(t-n T) \\
& N=S+I .
\end{aligned}
$$

This implies that the dynamics of the total population is given as

$$
\frac{d N}{d t}=(b-d) N-r d N^{2}-\alpha I-p \sum_{n=1}^{\infty} I\left(n T^{-}\right) \delta(t-n T)
$$

The logistic dynamics is the result of the assumption that the death rate is an increasing function of the total population in the form $d(1+r N)$, where $r$ is a small positive parameter. The following theorem describes the asymptotic behavior of the infective and susceptible classes.

Theorem 4: Consider the system of equations (16)-(18) with $b>0, \beta-(b+$ $\alpha)>0$ and all other parameters non-negative. We have: 
(1) If $p \geq 1-e^{-(\beta-b-\alpha) T}=p_{1}^{*}$ then the disease free equilibrium $(s, i)=$ $(1,0)$ always exists and it is globally asymptotically stable in the feasibility region $\mathcal{D}$. Moreover, $I(t) \rightarrow 0$ and $N(t) \rightarrow \frac{b-d}{r d}$ as $t \rightarrow \infty$.

(2) If $p<p_{1}^{*}$ then there exists a unique endemic periodic solution $\left(i^{*}, s^{*}\right)$ and this solution is globally asymptotically stable in $\mathcal{D}_{1}$. In this case $I(t)$ and $N(t)$ both converge to a positive periodic function, so the disease stays endemic in the population.

Proof: We proceed as in Section 2 and rewrite equations (16)-(18) in terms of fractions of infected and susceptible classes. Thus we obtain

$$
\begin{aligned}
& \frac{d s}{d t}=b(1-s)-(\beta-\alpha) s i+\sum_{n=1}^{\infty} p \frac{i\left(n T^{-}\right) s\left(n T^{-}\right)}{1-p i\left(n T^{-}\right)} \delta(t-n T) \\
& \frac{d i}{d t}=-(b+\alpha) i+\beta s i+\alpha i^{2}-\sum_{n=1}^{\infty} p \frac{i\left(n T^{-}\right)\left(1-i\left(n T^{-}\right)\right)}{1-p i\left(n T^{-}\right)} \delta(t-n T)
\end{aligned}
$$

with feasibility set $\mathcal{D}=\{(s, i) \mid s \geq 0, i \geq 0, s+i=1$.

We can see that equation (21) is the same as the one governing the behavior of $i(t)$ with exponential underlying population dynamics (7). Hence, our previous results are directly applicable. Thus we have that for $p \geq 1-e^{-(\beta-b-\alpha)}=p_{1}^{*}$ $i(t) \rightarrow 0$ as $t \rightarrow \infty$, while $i(t)$ tends to a positive periodic function when $p<p_{1}^{*}$. Next we examine the behavior of $I(t)$. The logistic dynamics of the total population in (19) guarantees the boundedness of $N$, so $i(t) \rightarrow 0$ implies that the total number of infective individuals $I(t)$ tends to 0 as well. This in turn implies that $N(t) \rightarrow \frac{b-d}{r d}$ (the positive equilibrium of (19) with $I=0$ ) as $t \rightarrow \infty$. Thus in this case we have only one threshold quantity that separates the asymptotic behavior of the infected and susceptible classes. In the case when $p<p_{1}^{*}$ we have that $i(t)$ tends to a positive endemic solution $i^{*}(t)$. Plugging in $I(t)=i^{*}(t) N(t)$ into (19) yields that $N(t)$ also tends to a positive periodic function. Figures 6 and 7 illustrate the behavior of the system for $p>p_{1}^{*}$ and $p<p_{1}^{*}$.
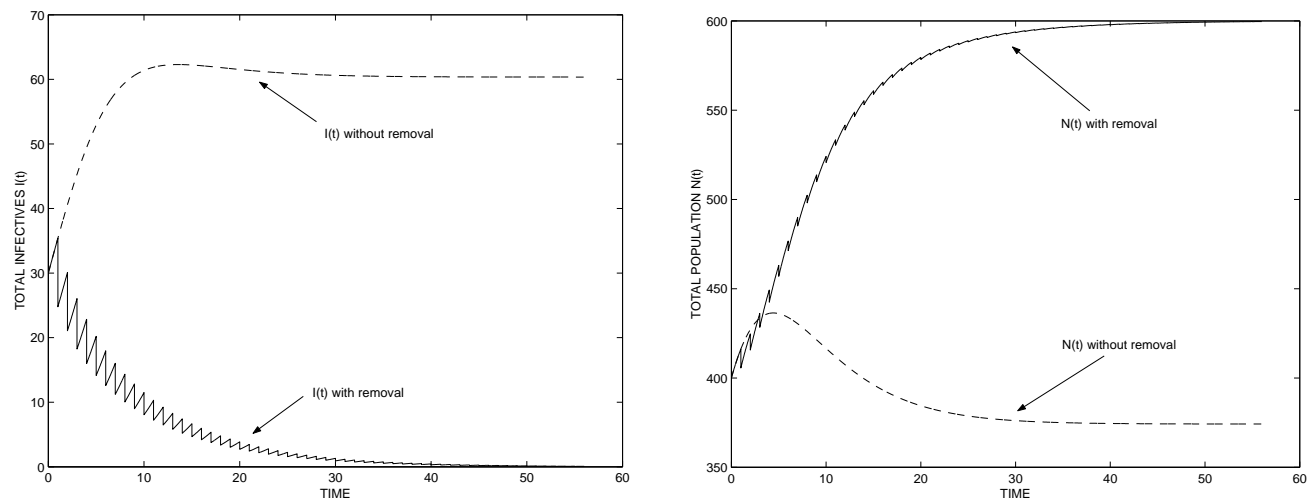

Fig. 6. Logistic example for $p>p_{1}^{*}$, infected class (left), total population (right) 

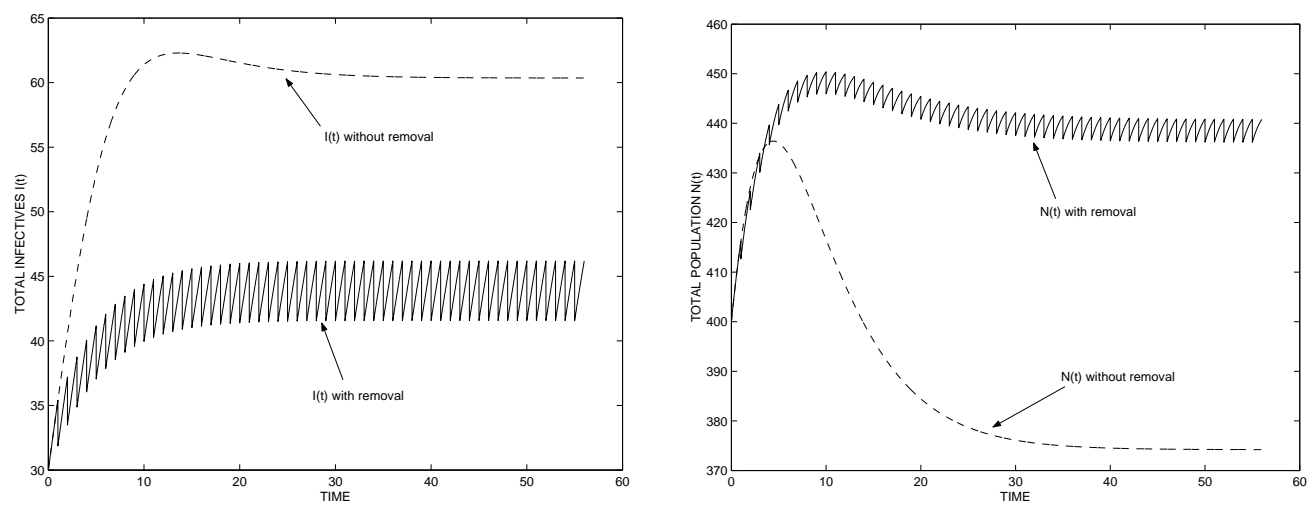

Fig. 7. Logistic example for $p<p_{1}^{*}$, infected class (left), total population (right)

\section{Acknowledgments}

This research was supported in part (G.A.P.) by the U.S. Air Force Office of Scientific Research under grant AFOSR F49620-03-1-0185.

The authors are grateful to Prof. Azmy Ackleh for several informative discussions on this problem.

\section{References}

[1] Z. Agur, B. Shulgin and L. Stone, Pulse Faccination Strategy in the SIR Epidemic Model, Bulletin of Mathematical Biology, 60 (1998), pp.1-26.

[2] Z. Agur, B. Shulgin and L. Stone, Theoretical Examination of the Pulse Vaccination Policy in the SIR Epidemic Model, Mathematical and Computer Modelling, 31 (2000), pp. 207-215.

[3] N.T. Bailey, The Mathematical Theory of Epidemics, Charles Griffin \& Company Limited, 1957.

[4] D.D. Bainov and P.S. Simeonov, Systems with Impulse Effect: stability, theory and applications, Ellis Horwood Limited, 1989.

[5] D.D. Bainov, V. Lakshmikantham and P.S. Simeonov, Theory of Impulsive Differentian Equations in Series in Modern Applied Mathematics, 6, World Scientific, 1989. Charles Griffin \& Company Limited, 1957.

[6] S. Busenberg and P. van den Driessche, Analysis of a disease transmission model in a population with varying size., J. Math. Biol., 28 (1990), pp. 257-270.

[7] A. d'Onofrio, Pulse Vaccination Strategy in the SIR Epidemic Model: Global Asymptotic Stable Eradication in Presence of Vaccine Failures, Mathematical and Computer Modeling, 36 (2002), pp. 473-489.

[8] A. d'Onofrio, Stability properties of pulse vaccination strategy in SEIR epidemic model, Mathematical Biosciences, 179 (2002), pp. 57-72. 
[9] M.W. Miller and J.E. Gross Chronic wasting disease in mule deer: disease dynamics and control, Journal of Wildlife Management, 65 (2001), pp. 205-215.

[10] J.A.P. Heesterbeek and M.G. Roberts Mathematical Models for Microparasites of WildlifeIn Ecology of Infectious Disease in Natural Populations, B. T. Grenfell and A. P. Dobson (eds), Cambridge: Cambridge University Press, 1995, pp. 90-122.

[11] H.W. Hethcote, H.W. Stech, H.W. and P.van den Driessche, Periodicity and stability in epidemic models: a survey, in S.N. Busenberg, K.L. Cooke (eds.) Differential Equations and Applications in Ecology, Epidemicand Populations Problems, New York: Academic Press (1981) pp.65-82

[12] H.W. Hethcote, A thousand and one epidemic models, Frontiers in Theoretical Biology, S.A. Levin (ed.), Lecture Notes in Biomathematics, 100, Springer, Berlin. 